\title{
The challenges of renal replacement therapy in Asia
}

\author{
Georgi Abraham
}

Asia covers a little less than a third of the earth's land area but is home to two-thirds of the world's inhabitants, distributed over 37 countries. These countries encompass lowincome nations and rapidly growing economies, in addition to diverse cultures, languages and religious beliefs. Asian countries also have varying burdens of noncommunicable disease because of this diversity.

Chronic kidney disease in Asia is predominantly due to diabetes and hypertension and is rising in prevalence as screening becomes more widespread. Glomerulonephritis is more common in Asia than in the Western world, perhaps because of infections and overcrowding. Although few Asian countries have renal replacement therapy (RRT) registries, the number of patients with end-stage renal disease (ESRD) is clearly growing in this region.

Universally reimbursed and equitable RRT programs are rare in Asia (Just PM et al. [2008] Nephrol Dial Transplant 23: 2365-2373) and most of these nations remain dependent on expensive imported technology for RRT. Private health care forms the backbone of RRT in the developing world; as a result, it is estimated that over $90 \%$ of patients with ESRD in South Asia die within months of diagnosis because they cannot afford treatment.

In India, which has a population of 1.1 billion, the age-adjusted incidence of ESRD is estimated at 229 per million. India spends 5.2\% of its annual gross domestic product on health care; the government contributes only $0.9 \%$ of the gross domestic product to health care (Abraham G et al. [2008] Perit Dial Int 28: 13-19). In Myanmar, RRT is available only in major cities and must be paid for by the patient. Japan, South Korea, Hong Kong, Taiwan, Singapore and some West Asian nations have RRT programs supported by their governments. Little is known about RRT in countries of the former Soviet Union or in war-torn Afghanistan.

Hemodialysis is the most common form of RRT in Asia, followed by peritoneal dialysis and
... . it is

estimated

that over $90 \%$

of patients

with ESRD in

South Asia die

within months

of diagnosis

because they

cannot afford

treatment.

G Abraham is

Professor of Medicine at Sri Ramachandra

University and

Madras Medical

Mission Hospital,

Chennai, India, and a Member of the ISN Council.

Competing interests

The author declared no

competing interests.

www.nature.com/clinicalpractice doi:10.1038/ncpneph0978 renal transplantation. Approximately 500,000 patients currently receive maintenance dialysis, most of whom are in China and Japan. In some countries (e.g. Japan and South Korea), home peritoneal dialysis is cheaper than hemodialysis. Strategies used to promote peritoneal dialysis include 'peritoneal dialysis first' policies, incentives to patients and payers, and the establishment of nongovernmental peritoneal dialysis centers (Li PK et al. [2007] Perit Dial Int 27 [Suppl 2]: S59-S61).

Renal transplantation practices vary from country to country depending upon social security coverage, legal status of brain death, religious beliefs and private-government partnerships. Government-appointed authorities oversee transplantation in some countries; in India, transplantation centers provide immunosuppressive therapy for free only in government hospitals. Infections are a common cause of morbidity and mortality after transplantation in Asia. Commercial transplantation has declined considerably as a result of media reports and government action.

Understaffing of the nephrology workforce, caused by a lack of training positions for aspiring doctors and the 'brain drain' to developed economies, is another major challenge facing RRT in Asia. South Asia, which has a population of 1.4 billion, possesses fewer than 1,300 nephrologists.

Asian economies are forecasted to reach the standards of developed nations in the next two to three decades, and the challenge of RRT can be met only by harnessing the growing financial, human and technological resources. The International Society of Nephrology has funded several screening and early detection programs for chronic kidney disease in developing Asian nations. Innovative public-private partnerships involving governments, nongovernmental organizations and philanthropists are needed to further raise awareness of kidney disease, facilitate early detection and prevention, and establish accessible RRT programs. 